\title{
AN ANSWER TO A QUESTION OF M. NEWMAN ON MATRIX COMPLETION
}

\author{
L. N. VASERSTEIN ${ }^{1}$
}

\begin{abstract}
Let $R$ be a principal ideal ring, $A$ a symmetric $t$-by- $t$ matrix over $R, B$ a $t$-by- $(n-t)$ matrix over $R$ such that the $t$-by- $n$ matrix $(A, B)$ is primitive. Newman [2] proved that $(A, B)$ may be completed (as the first $t$ rows) to a symmetric $n$-by- $n$ matrix of determinant 1 , provided that $1 \leq t \leq$ $n / 3$. He showed that the result is false, in general, if $t=n / 2$, and he asked to determine all values of $t$ such that $1 \leq t \leq n$ and the result holds. It is shown here that these values are exactly $t$ satisfying $1 \leq t<n / 2$.

Moreover, the result is proved for a larger (than the principal ideal rings) class of commutative rings, namely, for the rings satisfying the second stable range condition of Bass [1].

Also, it is observed that Theorems 2 and 3 of $[2$, p. 40] proved there for principal ideal rings are true for this larger class of rings, as well as the basic result of $[\mathbf{2}$, p. 39].
\end{abstract}

Statement of results. In this paper, "ring" means "commutative associative ring with 1".

We use the notation $\operatorname{sr}(R)[3,7]$. In particular, $\operatorname{sr}(R) \leq 1$ means that the following condition (the first stable range condition of Bass [1]) holds:

$(\operatorname{sr}(R) \leq 1)$. If $a_{1}, a_{2}$ are in $R$ and $a_{1} R+a_{2} R=R$, then $\left(a_{1}+a_{2} c\right) R=R$ for some $c$ in $R$.

This condition was discussed in detail in [6], where many examples are given. A. Roy showed me principal ideal rings with $\operatorname{sr}(R) \leq 1$ which are not semilocal. B. Magurn showed me Dedekind rings $R$ with $\operatorname{sr}(R) \leq 1$ which are not principal ideal rings. The following theorem gives more examples (including all Boolean rings $R$ with 1).

THEOREM 1. Suppose that there is a primitive polynomial $f(x)$ in one variable $x$ with integral coefficients such that $f(r)=0$ for every element $r$ of a ring $R$. Then $\operatorname{sr}(R) \leq 1$.

The second stable range condition, $\operatorname{sr}(R) \leq 2$, reads as follows:

$(\operatorname{sr}(R) \leq 2)$. If $a_{1}, a_{2}, a_{3}$ are in $R$ and $a_{1} R+a_{2} R+a_{3} R=R$, then $\left(a_{1}+a_{3} c_{1}\right) R+$ $\left(a_{2}+a_{3} c_{2}\right) R=R$ for some $c_{1}, c_{2}$ in $R$.

If $R$ is Noetherian of finite Krull dimension $\operatorname{dim}(R)$, then $\operatorname{sr}(R) \leq 1+\operatorname{dim}(R)$, by Bass $[\mathbf{1}]$. In particular, $\operatorname{sr}(R) \leq 1+\operatorname{dim}(R) \leq 1+1=2$ for each principal ideal ring $R$.

Here are more examples of rings $R$ with $\operatorname{sr}(R) \leq 2$ : The ring of integers in any number field or, more generally, any subring $R$ with 1 of any global field; any $R$ whose space of maximal ideals is Noetherian of dimension $\leq 1$; any finitely

Received by the editors June 4, 1985.

1980 Mathematics Subject Classification. Primary 18F25, 15 A33.

${ }^{1}$ Supported in part by the National Science and Guggenheim Foundations. 
generated algebra $R$ over any finite field with $\operatorname{dim}(R) \leq 2$ (see $[\mathbf{7}]$ ); the ring of real continuous functions on an interval or, more generally, on any topological space of dimension $\leq 1$ (see $[3]$ ); the ring of all continuous complex functions on a topological space of dimension $\leq 3$ (see $[\mathbf{3}]$ ).

For any ring $R$ and any natural numbers $t$ and $n$, let $M_{t, n} R$ denote the set of all $t$-by- $n$ matrices over $R$. A matrix in $M_{t, n} R$ is said to be unimodular if it has a left (when $t \leq n$ ) or right (when $t>n$ ) inverse in $M_{n, t} R$. This is equivalent for this matrix to be primitive, i.e. the ideal of $R$ generated by all maximal minors to be $R$.

Let $\mathrm{GL}_{n} R$ be the group of unimodular (i.e. invertible) matrices in $M_{n, n} R$, and let $\mathrm{SL}_{n} R$ denote the subgroup of matrices with determinant 1 .

THEOREM 2. Let $R$ be a ring, and let $t$ and $n$ be natural numbers such that $t \leq n$. If $\operatorname{sr}(R) \leq n-2 t+1$ and $t<n / 2$, then

$\left(R_{t, n}\right)$. For every symmetric matrix $A=A^{T}$ in $M_{t, t}$ and every matrix $B$ in $M_{t, n-t} R$ such that the matrix $(A, B)$ in $M_{t, n} R$ is unimodular, there is a symmetric matrix in $\mathrm{SL}_{n} R$ whose first $t$ rows form the given matrix $(A, B)$.

THEOREM 3. Let $R$ be a ring, and let $t$ and $n$ be natural numbers such that $t \leq n$. Then

(a) when $t=n$, the statement $\left(R_{t, n}\right)$ above is true if and only if the group $\mathrm{GL}_{1} R$ is trivial, i.e 1 is the only unit of $R$;

(b) when $t=n / 2$ is odd, $\left(R_{t, n}\right)$ is true if and only if $r^{4}=r^{2}$ for all $r$ in $R$ and $2 R=0$;

(c) when $t=n / 2$ is even, $\left(R_{t, n}\right)$ is true if and only if $\left(r^{2}-2 r\right)\left(r^{2}-1\right)=0$ for all $r$ in $R$;

(d) when $n / 2<t<n,\left(R_{t, n}\right)$ is true if and only if $r^{2}=r$ for all $r$ in $R$, i.e. $R$ is a Boolean ring.

Combining Theorems 2 and 3 we obtain the following complete answer to the open question (c) of [2, p. 45].

COROLlaRY. If $\operatorname{sr}(R) \leq 2$ (for example, $R$ is a principal ideal ring) then the statement $\left(R_{t, s}\right)$ holds, provided that $1 \leq t<n / 2$. If $(r-2)\left(r^{4}-r^{2}\right) \neq 0$ for some $r$ in $R$, then the statement $\left(R_{t, n}\right)$ never holds for $n / 2 \leq t<n$.

However, Theorem 3 justifies a modification of the question of Newman for $t \geq n / 2$. For example, $\left(R_{n, n}\right)$ becomes true if we replace $\mathrm{SL}_{n} R$ in it by $\mathrm{GL}_{n} R$. The following theorem answers the modified question for all $t$.

THEOREM 4. The statement $\left(R_{t, n}\right)$ with $\mathrm{SL}_{n} R$ replacea by $\mathrm{GL}_{n} R$ is true for all $t$ and $n$ such that $t \leq n$ if and only if $\operatorname{sr}(R) \leq 1$.

REMARK. Let $\operatorname{sr}(R) \leq 2$. Then, by $[\mathbf{4}, \mathbf{5}]$, for any natural numbers $t$ and $n$ such that $t \leq n$ and any matrix $C$ in $M_{t, t} R$, the symplectic group

$$
\mathrm{Sp}_{2 n} R=\left\{D \in \mathrm{SL}_{2 n} R: D^{T}\left(\begin{array}{cc}
0 & 1_{n} \\
-1_{n} & 0
\end{array}\right) D=\left(\begin{array}{cc}
0 & 1_{n} \\
-1_{n} & 0
\end{array}\right)\right\}
$$

acts transitively on the matrices $(A, B)$ in $M_{t, 2 n} R$ with $A, B$ in $M_{t, n} R$ and $A B^{T}-$ $B A^{T}=C$. 
Taking here $C=0$ and $t=1$ (respectively, $t=n$ ), we obtain Theorem 2 (resp., Theorem 3) of [2], extended from principal ideal rings $R$ to any $R$ with $\operatorname{sr}(R) \leq 2$.

PROOF OF THEOREM 1 . Let $d$ be the degree of the polynomial $f(x)$. Then for any maximal ideal $m$ of $R$ the polynomial vanishes on the field $R / m$, hence $\operatorname{card}(R / m) \leq d$. Therefore $r^{d !} \equiv r(\bmod m)$ for every element $r$ in $R$ and any maximal ideal $m$ of $R$.

Let $\left(a_{1}, a_{2}\right)$ be an arbitrary unimodular row in $M_{1,2} R$. We set $c=a_{1}^{d !}-1$. The claim is that $\left(a_{1}+a_{2} c\right) R=R$. It suffices to show that the left-hand side is not contained in any maximal ideal $m$ of $R$. Passing to the factor ring $R / m$, we see that is suffices to prove the claim in the case when $R$ is a field. In this case,

$$
a_{1}+a_{2} c= \begin{cases}a_{1} & \text { if } a \neq 0 \text { (because then } c=0) \\ a_{2} & \text { if } \left.a_{1}=0 \text { (because then } c=-1\right) .\end{cases}
$$

So $a_{1}+a_{2} c \neq 0$, hence $\left(a_{1}+a_{2} c\right) R=R$.

PROOF OF THEOREM 2. We will use the following two lemmas:

LEMMA 1 (SEE $[\mathbf{3}]$ ). For any natural numbers $d, s, t$ the condition $\operatorname{sr}(R) \leq d$ is equivalent to the following condition: For any matrices $A$ in $M_{s, d+s-1} R$ and $B$ in $M_{s, t} R$ such that $(A, B)$ in $M_{s, d+s-1+t} R$ is unimodular there is a matrix $C$ in $M_{t, r d+s-1} R$ such that the matrix $A+B C$ in $M_{s, d+s-1} R$ is unimodular.

LEMMA 2 (SEE $[\mathbf{1}, \mathbf{5}, \mathbf{7}])$. For any $s, t$ with $t \geq s+\operatorname{sr}(R)$, the group $\mathrm{SL}_{t} R$ acts transitively on the set of unimodular matrices in $M_{s, t} R$; if $\operatorname{sr}(R) \leq 2$, then the condition $t \geq s+\operatorname{sr}(R)$ above can be replaced by $t \geq s+1$.

REMARK. Lemma 2 can be easily proved by induction on $s$. After this, it is not hard to prove Lemma 1 also by induction on $s$. Of course, Lemma 2 is a trivial consequence of Lemma 1 . Lemma 2 generalizes the basic result of [2, p. 39].

Now we are ready to prove Theorem 2 . Let $\operatorname{sr}(R) \leq n-2 t+1$ and let $A$ and $B$ be as in $\left(R_{t, n}\right)$.

Case $1 . B$ has the form $\left(1_{t}, 0\right)$, where $1_{t}$ is the identity matrix in $\mathrm{SL}_{t} R$ and 0 is the zero matrix in $M_{t, n-2 t} R$. Then for any matrix $X=X^{T}$ in $M_{n-2 t, n-2 t} R$ the matrix

$$
Y=\left(\begin{array}{ccc}
A & 1_{t} & 0 \\
1_{t} & 0 & 0 \\
0 & 0 & X
\end{array}\right) \quad \text { in } M_{n, n} R
$$

is symmetric, its first $t$ rows form the given matrix $(A, B)=\left(A, 1_{t}, 0\right)$, and $\operatorname{det}(Y)=$ $(-1)^{t} \operatorname{det}(X)$. Choosing $X$ with $\operatorname{det}(X)=(-1)^{t}$ (a diagonal matrix will do), we complete our proof in Case 1.

Case 2. $B$ is unimodular. Then by Lemma 2 there is a matrix $C$ in $\mathrm{SL}_{n-t} R$ such that $B C=\left(1_{t}, 0\right)$. By Case 1 , there is a symmetric matrix $Y$ in $\mathrm{SL}_{n} R$ of the form $Y=\left(\begin{array}{cc}A & B C \\ * & *\end{array}\right)$. Then the symmetric matrix

$$
\left(\begin{array}{cc}
1_{t} & 0 \\
0 & C^{-1}
\end{array}\right)^{T} Y\left(\begin{array}{cc}
1_{t} & 0 \\
0 & C^{-1}
\end{array}\right) \quad \text { in } \mathrm{SL}_{n} R
$$

is of the form $\left(\begin{array}{ll}A & B \\ * & *\end{array}\right)$. 
General case. By Lemma 1 , there is a matrix $D$ in $M_{t, n-t} R$ such that $B+A D$ in $M_{t, n-t} R$ is unimodular. By Case 2 , there is a symmetric matrix $Y$ in $\mathrm{SL}_{n} R$ of the form $Y=\left(\begin{array}{cc}A & B+A D \\ * & *\end{array}\right)$. Then the symmetric matrix

$$
\left(\begin{array}{cc}
1_{t} & 0 \\
-D^{T} & 1_{n-t}
\end{array}\right) Y\left(\begin{array}{cc}
1_{t} & -D \\
0 & 1_{n-t}
\end{array}\right) \quad \text { in } \mathrm{SL}_{n} R
$$

is of the form $\left(\begin{array}{ll}A & B \\ *\end{array}\right)$.

ProOF OF THEOREM 3. Part (a) is evident, so let $n / 2 \leq t<n$.

LEMMA 3 . The condition $\left(R_{t, 2 t}\right)$ implies that $b^{2} \equiv(-1)^{t}(\bmod a R)$ for every unimodular row $(a, b)$ in $M_{1,2} R$.

Proof. We set $A=\left(\begin{array}{ll}0 & 0 \\ 0 & a\end{array}\right)$ and $B=\left(\begin{array}{cc}1_{t-1} & 0 \\ 0 & b\end{array}\right)$ (both in $M_{t, t} R$; when $t=1$, $(A, B)=(a, b))$. Let $Y$ be a symmetric matrix in $\mathrm{SL}_{2 t} R$ of the form $Y=\left(\begin{array}{cc}A & B \\ * & *\end{array}\right)$. Then $1=\operatorname{det}(Y)=(-1)^{t-1} \operatorname{det}\left(\begin{array}{ll}a & b \\ b & d\end{array}\right)=(-1)^{t}\left(b^{2}-a d\right)$, where $d$ is the $(2 t, 2 t)$-entry of the matrix $Y$. The lemma is proved.

Let us now prove the "only if" parts of (b)-(d) of Theorem 3. We assume $\left(R_{t, n}\right)$ and want to obtain certain conclusions about the ring $R$.

(b) Taking $(a, b)=(0,1)$ in Lemma 3 , we conclude that $2=0$ in $R$, i.e. $2 R=0$. For every maximal ideal $m$ of $R$ and any $b$ in $R$ outside of $m$ we can find an element $r$ in $R$ such that $r b-1 \in m$. Applying Lemma 3 to $(a, b)=(r b-1, b)$, we obtain that $b^{2}-1 \in m$.

Therefore $\operatorname{card}(B / m)=2$ for every maximal ideal $m$ of $R$, hence $r^{2}-r \in m$ for every $m$, i.e. $r^{2}-r \in \operatorname{rad}(R)$ for every $r$ in $R$, where $\operatorname{rad}(R)$ is the Jacobson radical of $R$, i.e. the intersection of all maximal ideals.

In particular, $1+r^{2}-r=1+r^{2}+r \in \mathrm{GL}_{1} R$ for every $r$ in $R$. By Lemma 3 with $(a, b)=\left(0,1+r+r^{2}\right)$, we obtain that $\left(1+r+r^{2}\right)^{2}=1+r^{2}+r^{4}=1$, hence $r^{2}=r^{4}$ for all $r$ in $R$.

(c) For any maximal ideal $m$ of $R$ and any $b$ outside of $m$ we have $r b-1 \in m$ for some $r$ in $R$. By Lemma 3 with $(a, b)=(r b-1, b)$, we obtain $b^{2} \equiv 1(\bmod m)$. So $\operatorname{card}(R / m)=2$ or 3 for every maximal ideal $m$ of $R$. By Lemma 3 with $(a, b)=\left(0,1+r^{3}-r\right)$, we obtain that $\left(1+r^{3}-r\right)^{2}=1$ for all $r$ in $R$. Setting here $r=2$, we have $48=0$ in $R$. So the ring $R$ splits into the direct product of its 2 -primary component $3 R$ and its 3 -primary component $8 R$.

In $3 R$ we have $r^{2}-r \in \operatorname{rad}(3 R)$, so $\left(1-r^{2}+r\right)^{2}=1$, hence

$$
\left(1+r-r^{2}\right)^{2}-1=\left(r^{2}-r\right)\left(r^{2}-r-2\right)=\left(r^{2}-2 r\right)\left(r^{2}-1\right)=0
$$

for all $r$ in $3 R$.

In the ring $8 R$ we have $r^{3}-r \in \operatorname{rad}(8 R)$, so $\left(1 \pm\left(r^{3}-r\right)\right)^{2}=1$, hence $4\left(r^{3}-r\right)=$ $\left(r^{3}-r\right)=0$, so $(r-2)\left(r^{3}-r\right)=\left(r^{2}-2 r\right)\left(r^{2}-1\right)=0$ for all $r$ in $8 R$.

Thus, $\left(r^{2}-2 r\right)\left(r^{2}-1\right)=0$ for all $r$ in $R$.

(d) For an arbitrary unit $u$ of $R$ we pick some $X=X^{T}$ in $\mathrm{GL}_{2 t-n} R$ with $\operatorname{det}(X)=u$ and define $A=\left(\begin{array}{ll}X & 0 \\ 0 & 0\end{array}\right)$ in $M_{t, t} R$. We set $B=\left(\begin{array}{c}0 \\ 1_{n-t}\end{array}\right)$ in $M_{t, n-t} R$. If $\left(\begin{array}{cc}A & B \\ *^{*} & *\end{array}\right)=Y$ is a symmetric matrix in $\mathrm{SL}_{n} R$, then

$$
1=\operatorname{det}(Y)=(-1)^{n-t} \operatorname{det}(X)=(-1)^{n-t} u,
$$

hence $u=(-1)^{n-t}$. 
Thus, the group $\mathrm{GL}_{1} R$ is trivial. In particular, $2=0$ in $R$.

Evidently, $\left(R_{t, n}\right)$ implies $\left(R_{t-(2 t-n), n-(2 t-n)}\right)=\left(R_{n-t, 2(n-t)}\right)$. By (b) and (c) proved above, $r^{4}-r^{2}=0$ when $n-t$ is odd and $\left(r^{2}-2 r\right)\left(r^{2}-1\right)=0$ when $n-t$ is even, for all $r$ in $R$. Since $2 R=0, r^{4}=r^{2}$ for all $r$ in all cases.

Since $\mathrm{GL}_{1} R$ is trivial, $\operatorname{rad}(R)=0$. So $r^{4}-r^{2}=\left(r^{2}-r\right)^{2}=0$ implies that $r^{2}-r=0$ for all $r$ in $R$.

Now we have the "if" parts of (b)-(d) to prove. So we assume that the appropriate polynomials vanish on $R$, and we want to prove $\left(R_{t, n}\right)$.

By Theorem $1, \operatorname{sr}(R) \leq 1$. Let $(A, B)$ be as in $\left(R_{t, n}\right)$. If $t=n / 2$ (cases (b) and (c)), then, by Lemma $1, B-A C \in \mathrm{GL}_{t} R$ for some $C$ in $M_{t, t} R$. So the matrix

$$
\begin{aligned}
Y & =\left(\begin{array}{cc}
1 & 0 \\
C^{T} & 1
\end{array}\right)\left(\begin{array}{cc}
A & B-A C \\
(B-A C)^{T} & 0
\end{array}\right)\left(\begin{array}{ll}
1 & C \\
0 & 1
\end{array}\right) \\
& =\left(\begin{array}{cc}
A & B \\
B^{T} & B^{T} C+C^{T} B-C^{T} A C
\end{array}\right)
\end{aligned}
$$

is symmetric with $\operatorname{det}(Y)=(-1)^{t} \operatorname{det}(B-A C)^{2}$, where 1 stands for $1_{t}$.

In case (b), the identity $r^{4}=r^{2}$ gives that $u^{2}=1$ for every unit $u$ of $R$. The identity $2 r=0$ gives that $(-1)^{t}=-1=1$. So $\operatorname{det}(Y)=(-1)^{t} \operatorname{det}(B-A C)^{2}=1$.

In case (c) the identity $\left(r^{2}-2 r\right)\left(r^{2}-1\right)=0$ gives (when $r=3$ ) that $24=0$ in $R$. So $R$ splits into the direct product of a 2-primary component $3 R$ and a 3-primary component $8 R$. On $3 R, u^{2}-2 u$ is a unit for any unit $u$, so the identity gives tht $u^{2}=1$. On $8 R$, setting $r=u-1$, we obtain that $u^{2}(u-1)(u-2)=0$, hence $(u-1)(u-2)=u^{2}-1=0$ for any unit $u$. Thus, $u^{2}=1$ on $R$ for any unit $u$. In particular, $\operatorname{det}(Y)=\operatorname{det}(B-A C)^{2}=1$.

In case (d) our statement follows from Theroem 4 (which will be proved in the next section), because $\mathrm{GL}_{1} R$ is trivial for any Boolean ring $R$.

PROOF OF THEOREM 4. First, we want to prove that $\operatorname{sr}(R) \leq 1$, assuming that the statement $\left(R_{t, n}\right)$ with $\mathrm{SL}_{n} R$ replaced by $\mathrm{GL}_{n} R$ is true for some $t, n$ such that $3 / 2 \leq n / 2 \leq t<n$.

Let $a, b$ be in $R$. We set

$$
\begin{aligned}
& (A, B)=X_{2}=\left(\begin{array}{llll}
a & 1 & 0 & 0 \\
1 & b & 1 & 0
\end{array}\right) \quad \text { when } t=2=n / 2 \\
& (A, B)=X_{t}=\left(\begin{array}{ccc}
0 & 0 & 1_{n-t-2} \\
0 & X_{2} & 0
\end{array}\right) \quad \text { when } n=2 t>4 \\
& (A, B)=\left(\begin{array}{cc}
1_{2 t-n} & 0 \\
0 & X_{n-t}
\end{array}\right) \quad \text { when } n>2 t \geq 4
\end{aligned}
$$

If $Y=\left(\begin{array}{cc}A & B \\ * & *\end{array}\right)$ is a symmetric matrix in $\mathrm{GL}_{n} R$, then

$$
\mathrm{GL}_{1} R \ni \operatorname{det}(Y)=(-1)^{n-t} \operatorname{det}\left(\begin{array}{lll}
a & 1 & 0 \\
1 & b & 1 \\
0 & 1 & y
\end{array}\right)=(-1)^{n-t}(y(a b-1)-a),
$$

where $y$ is the $(n, n)$ th entry of $Y$. 
Thus, for any $a, b$ in $R$, there is some $y$ in $R$ such that $y(a b-1)-a$ is a unit. Let us show that this implies that $\operatorname{sr}(R) \leq 1$ (as matter of fact, this is equivalent to $\operatorname{sr}(R) \leq 1)$. Let $\left(a_{1}, a_{2}\right)$ in $M_{1,2} R$ be unimodular. Then $a_{1} b_{1}+a_{2} b_{2}=1$ for some $b_{1}, b_{2}$ in $R$. As above, we find $y$ in $R$ such that $y\left(a_{1} b_{1}-1\right)-a_{1} \in \mathrm{GL}_{1} R$. Since $a_{1} b_{1}-1=-a_{2} b_{2}$, we have $y a_{2} b_{2}+a_{1} \in \mathrm{GL}_{1} R$. So $a_{1}+a_{2} c \in \mathrm{GL}_{1} R$ for $c=y b_{2}$.

Assume now tht $\operatorname{sr}(R) \leq 1$. We have to prove $\left(R_{t, n}\right)$ with $\mathrm{SL}_{n} R$ replaced by $\mathrm{GL}_{n} R$, where $t \leq n$. When $t \leq n / 2$, we are done by Theorem 2 . The case $t=n$ is trivial. So we assume that $n / 2 \leq t \leq n-1$.

Let $(A, B)$ be as in $\left(R_{t, n}\right)$. If $t=n / 2$, then by Lemma $1, B-A C \in \mathrm{GL}_{t} R$ for some $C$ in $M_{t, t} R$ and we are done by a computation in a previous section.

Let now $n / 2<t<n$. When we replace $(t, n)$ by $(t+1, n+1)$, we make our statement (the modified $\left(R_{t, n}\right)$ which we are proving) stronger. So. after a few such replacements, we can assume that $n$ is divisible by $n-t$. Set $k=n /(n-t)-1$.

Note that $(A, B)$ can be completed to a symmetric matrix in $\mathrm{GL}_{n} R$ if and only if $\left(D A D^{T}, D(B+A C)\right.$ ) can, where $C$ is a matrix in $M_{t, t} R$ and $D \in \mathrm{GL}_{t} R$ (compare with the proof of Theorem 2). Since $\operatorname{sr}(R) \leq 1$, Lemma 1 and 2 allow us to find $C, D$ satisfying $D(B+A C)=\left(\begin{array}{c}0 \\ 1_{n-t}\end{array}\right)$ in $M_{t, n-t} R$. So we can assume that $B=\left(\begin{array}{c}0 \\ 1_{n-t}\end{array}\right)$ from the beginning.

Repeating this argument, we can assume that the matrix $(A, B)$ in $M_{t, n} R$ has $D_{1}, D_{2}, \ldots, D_{k+1}$ along the main diagonal with $D_{i}$ in $M_{n-t, n-t} R ; 1_{n-t}$ on the line above the main diagonal and on the line below the diagonal; 0 elsewhere.

Applying Lemma 4 below with $s=n-t$ and $U_{k}=1_{s}$ to the matrix $Y=\left(\begin{array}{cc}A & B \\ B^{T} & X\end{array}\right)$ in $M_{n, n} R$, we see that $\operatorname{det}(Y)=\operatorname{det}(V+W X)$ for some $V, W$ in $M_{s, s} R$ with unimodular $(V, W)$ and $V W^{T}=W V^{T}$. Using Lemma 5 below, we find a symmetric $X$ such that $Y \in \mathrm{GL}_{n} R$.

Thus, Theorem 4 is reduced to the following two lemmas.

LEMMA 4. Let $s, k$ be natural numbers, $R$ a ring, $D_{1}, \ldots, D_{k+1}, U_{1}, \ldots, U_{k}$ matrices in $M_{s, s} R$ such that $U_{i}=1_{s}$ for $i<k$ and all $D_{i}$ are symmetric. Let $Y$ be the matrix in $M_{s k+s, s k+s} R$ with $D_{i}$ along the main diagonal, $1_{s}$ along the line below the diagonal, $U_{i}$ along the line above the diagonal, and 0 elsewhere. Then $\operatorname{det}(Y)=\operatorname{det}\left(V U_{i}+W D_{k+1}\right)$ for some matrices $V, W$ in $M_{s, s} R$ with unimodular $(V, W)$ and symmetric $V W^{T}$.

ProOF. We proceed by induction on $k$. When $k=1, Y=\left(\begin{array}{cc}D_{1} & U_{1} \\ 1_{s} & D_{2}\end{array}\right)$, so

$$
\operatorname{det}(Y)=\operatorname{det}\left(\left(\begin{array}{cc}
1_{s} & -D_{1} \\
0 & 1_{s}
\end{array}\right) Y\right)=\operatorname{det}\left(\begin{array}{cc}
0 & U_{1}-D_{1} D_{2} \\
1_{s} & D_{2}
\end{array}\right)=\operatorname{det}\left(D_{1} D_{2}-U_{1}\right)
$$

so we can take $V=D_{1}$ and $W=-1_{s}$.

Suppose now that $k>1$. Using $1_{s}$ in the last $s$ rows of $Y=F\left(D_{1}, \ldots, D_{k+1} ; U_{k}\right)$, we can eliminate the term $D_{k+1}$ by column addition operations over $R$. Then it is clear that $\operatorname{det}(Y)=\operatorname{det}\left(F\left(D_{1}, \ldots, D_{k-1}, D_{k} D_{k-1}-U_{k} ; D_{k+1}\right)\right)$.

By the induction hypothesis, the last determinant has the form

$$
\operatorname{det}\left(V^{\prime} D_{k+1}+W^{\prime}\left(D_{k} D_{k+1}-U_{k}\right)\right)
$$


with unimodular $\left(V^{\prime}, W^{\prime}\right)$ and symmetric $V W^{T}$. But $V^{\prime} D_{k+1}+W^{\prime}\left(D_{k} D_{k+1}-U_{k}\right)=$ $V U_{k}+W D_{k+1}$ with

$$
(V, W)=\left(-W^{\prime}, V^{\prime}+W^{\prime} D_{k}\right)=\left(-W^{\prime}, V^{\prime}\right)\left(\begin{array}{cc}
1_{s} & -D_{k} \\
0 & 1_{s}
\end{array}\right)
$$

which is unimodular, because so is $\left(V^{\prime}, W^{\prime}\right)$. Moreover, $W V^{T}=-\left(V^{\prime}+W^{\prime} D_{k}\right) W^{\prime T}$ is symmetric, because so are $D_{k}$ and $V^{\prime} W^{\prime T}$.

LEMMA 5. Let $R$ be a ring with $\operatorname{sr}(R) \leq 1$. Then for any natural number $s$ and any matrices $V$ and $W$ in $M_{s, s} R$ such that $(V, W)$ in $M_{s, 2 s} R$ is unimodular and $V W^{T}=W V^{T}$ there is a matrix $X=X^{T}$ in $M_{s, s} R$ such that $V+W X \in \mathrm{GL}_{s} R$.

PROOF. We proceed by induction on $s$. When $s=1$, our conclusion coincides with our condition $\operatorname{sr}(R) \leq 1$. Let now $s \geq 2$.

By Lemma 1 and 2, there are matrices $C$ in $M_{s, s} R$ and $D$ in $\mathrm{GL}_{s} R$ such that $D(V+W C)=1_{s}$. Let $X_{1}$ be the symmetric matrix in $M_{s, s} R$ with the same first column as $C$ and with all entries outside the first column and row equal 0 . Then $D\left(V+W V_{1}\right)=\left(\begin{array}{cc}1 & u \\ 0 & V^{\prime}\end{array}\right)$ with some $u$ in $M_{1, s-1} R$ and $V^{\prime}$ in $M_{s-1, s-1} R$. We write $D W=\left(\begin{array}{cc}* & w \\ v & W^{\prime}\end{array}\right)$ with $v, w^{T}$ in $M_{s-1,1} R$ and $W^{\prime}$ in $M_{s-1, s-1} R$.

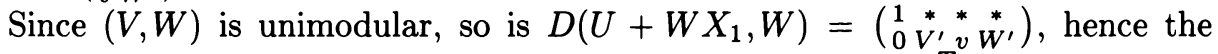
matrix $\left(V^{\prime}, v, W^{\prime}\right)$ in $M_{s-1,2 s-1} R$ is also unimodular. Since $W V^{T}$ is symmetric, so is

$$
D W\left(D\left(V+W X_{1}\right)\right)^{T}=\left(\begin{array}{cc}
* & w \\
v & W^{\prime}
\end{array}\right)\left(\begin{array}{cc}
1 & 0 \\
u^{T} & V^{\prime T}
\end{array}\right)=\left(\begin{array}{cc}
* & w V^{\prime T} \\
v+W^{\prime} u^{T} & W^{\prime} V^{\prime T}
\end{array}\right)
$$

hence $v+W^{\prime} u^{T}=V^{\prime} w^{T}$ and $W^{\prime} V^{\prime T}=V^{\prime} W^{\prime T}$. Since $\left(V^{\prime}, v, W^{\prime}\right)$ is unimodular and $v=V^{\prime} w^{T}-W^{\prime} u^{T}$, we conclude that $\left(V^{\prime}, W^{\prime}\right)$ is unimodular.

By the induction hypothesis, $V^{\prime}+W^{\prime} X^{\prime} \in \mathrm{GL}_{s-1} R$ for some symmetric matrix $X^{\prime}$ in $M_{s-1, s-1} R$. We set $X=X_{1}+\left(\begin{array}{cc}0 & 0 \\ 0 & X^{\prime}\end{array}\right)=X^{T}$ in $M_{s, s} R$. Then

$$
\begin{aligned}
D(V+W X) & =D\left(V+W X_{1}\right)+D W\left(\begin{array}{cc}
0 & 0 \\
0 & X^{\prime}
\end{array}\right) \\
& =\left(\begin{array}{cc}
1^{*} & \\
0 & V^{\prime}
\end{array}\right)+\left(\left(\begin{array}{cc}
* & * \\
* & W^{\prime}
\end{array}\right)\left(\begin{array}{cc}
0 & 0 \\
0 & X^{\prime}
\end{array}\right)\right) \\
& =\left(\begin{array}{cc}
1 & * \\
0 & V^{\prime}+W^{\prime} X^{\prime}
\end{array}\right) \in \mathrm{GL}_{s} R,
\end{aligned}
$$

hence $V+W X \in \mathrm{GL}_{s} R$.

\section{REFERENCES}

1. H. Bass, K-theory and stable algebra, Inst. Hautes Études Sci. Publ. Math. 22 (1964), 485-544.

2. M. Newman, Matrix completion theorems, Proc. Amer. Math. Soc. 94 (1985), 39-45.

3. L. N. Vaserstein, The stable range of rings and dimension of topological spaces, Funktsional. Anal. i Prilozhen. 5 (1971), 17-27 (translated in Functional Anal. Appl.).

4. Stabilization for unitary and orthogonal groups over a ring with involution, Mat. Sb. 81 (1970), 328-352 (translated in Math. USSR-Sb. 10). 
5. __ Stabilization for the classical groups over rings, Mat. Sb. 93 (1974), 268-295 (translated in Math. USSR-Sb. 22).

6. , Bass's first stable range conditions, J. Pure Appl. Algebra 34 (1984), 319-330.

7. L. N. Vaserstein and A. A. Suslin, Serre's problem on projective modules over polynomial rings and algebraic K-theory, Izv. Akad. Nauk SSSR Ser. Mat. 40 (1976), 993-1054 (translated in Math. USSR-Izv. 10).

Department of Mathematics, Pennsylvania State University, University PARK, PENNSYlVANiA 16802

School of Mathematics, Institute for Advanced Study, Princeton, New JERSEY 08540 (Current address) 